\title{
Le devenir phonétique des formes rédupliquées : réduplication, tons lexicaux et intonation en na (naxi)
}

\begin{abstract}
Alexis Michaud" et Jacqueline Vaissière ${ }^{\text {*** }}$
REFERENCES DE PUBLICATION : revue Faits de langues, n²9, pp. 23-35 (2007).

INTRODUCTION

Les langues d'Asie du Sud et du Sud-Est présentent une grande variété de phénomènes de réduplication, comme en témoignent par exemple l'étude d'Anvita Abbi 1997 et, dans le présent volume, celles de Guillaume Jacques, Annie Montaut, Marie-Claude Paris, Alice Vittrant et Françoise Robin, et Elizabeth Zeitoun. Le na, aussi appelé naxi ou moso, langue sino-tibétaine parlée à la frontière du Yunnan et du Sichuan (Chine), ne fait pas exception à ce constat typologique. Un argument phonologique paraît délimiter sans ambiguïté deux ensembles parmi les formes rédupliquées de cette langue qui possède des tons lexicaux (Haut, Moyen, Bas, et un ton montant, rare dans le lexique). En effet, un phénomène régulier de changement tonal a lieu dans deux cas (réduplication de monosyllabes portant un ton Haut ou un ton Bas) et non dans les autres.

Notre travail vise à montrer la proximité phonétique qui existe entre les formes avec et sans changement catégoriel de ton, proximité qui suggère que les deux ensembles de formes rédupliquées pourraient avoir pour origine commune une réduplication à l'identique : l'évolution ultérieure de deux des schémas serait due au figement de tendances phonétiques, dont l'effet demeure observable en synchronie. La variation phonétique de certains tons aurait été réinterprétée comme une différence phonologique de catégorie tonale. Une réflexion est proposée au sujet des conditions historiques d'une telle phonologisation. L'hypothèse proposée est que les composés rédupliqués auraient, à un certain stade, été porteurs d'un ton de mot, plutôt que d'un ton sur chacune de leurs syllabes. D'autres exemples de schèmes tonals inattendus sur des disyllabes sont observés dans la langue na, et peuvent être interprétés dans le même sens.
\end{abstract}

* Langues et Civilisations à Tradition Orale (UMR 7107), CNRS / Sorbonne / Sorbonne Nouvelle et Laboratoire de Phonétique et Phonologie (UMR 7018), CNRS/ Sorbonne Nouvelle. Courriel : alexis.michaud@vjf.cnrs.fr

** Laboratoire de Phonétique et Phonologie (UMR 7018), CNRS/ Sorbonne Nouvelle. Courriel : jacqueline.vaissiere@univ-paris3.fr 
L'étude de cette question est classiquement divisée en un exposé des faits phonologiques et des hypothèses, un volet expérimental, et une discussion de portée plus générale.

\section{LES FAITS NA (NAXI)}

Les deux dialectes étudiés ici sont le parler de la ville de Lijiang 丽江 et des environs, ci-après "dialecte de Lijiang ", homogène en ce qui concerne la réalisation tonale des schémas de réduplication, et le parler du village de /fỵ $\mathrm{k}^{\mathrm{h}} \overline{\mathrm{o}} /$ (丽江奉科乡善美行政村恒可二组; ci-après Fèng Kē). En l'absence d'indication contraire, les exemples proviennent du premier de ces dialectes, dont le système phonémique est décrit en détail par Michailovsky et Michaud 2006. Les données ont été recueillies sur place par le premier auteur de cet article, au cours de trois séjours de trois mois (en 2002, 2004 et 2006).

Le système tonal du na de Lijiang, relativement simple, comprend trois tons ponctuels : Haut, Moyen, Bas, transcrits par les diacritiques $/{ }^{\prime} /, / \%, /$. A Fèng $\mathrm{Ke}, \mathrm{il}$ existe un ton lexical supplémentaire : un contour montant. Dans les deux dialectes, des tons modulés apparaissent lors de l'ellipse d'une syllabe, dont seul demeure le ton, qui s'associe alors à la syllabe qui précède (phénomène décrit par Michaud 2006b). Aucun interdit ne pèse sur les séquences tonales : toute la combinatoire est attestée, sur les mots disyllabiques (Haut + Haut, Bas + Haut, Moyen + Bas, etc.) comme dans le fil de l'énoncé. De nombreux exemples de réduplication de mots lexicaux s'observent dans la langue courante (dialogues et récits), réduplication qui suit des schémas réguliers : dans certains (types $\mathrm{A}>$ $\mathrm{AA}$ et $\mathrm{AB}>\mathrm{AABB}$ ), un changement catégoriel de ton a lieu, dans d'autres non (type $\mathrm{AB}>\mathrm{ABAB}$ ). (Selon une convention répandue, dans les expressions $\mathrm{AA}$, $\mathrm{AABB}$ et $\mathrm{ABAB}$, chaque lettre désigne une syllabe : ainsi, /á lāa/ « s'affronter », issu de la réduplication du verbe /lá/ «frapper», sera décrit comme réduplication $A>A A$. Le symbole > signifie ici se réduplique en.) La comparaison des réalisations phonétiques de ces deux ensembles nous paraît fournir des indices révélateurs quant à leur origine historique. Avant d'aborder cette question phonétique/phonologique, qui constitue le cœur de l'article, un rapide panorama donnera une idée du rôle joué par ces formes dans la morphosyntaxe du na.

\subsection{Bref panorama sémantique des phénomènes de réduplication en na}

Dans cette langue, qui ne possède presque aucune morphologie verbale, la réduplication remplit un rôle de dérivation morphologique. La réduplication peut exprimer la répétition d'une action, la prolongation d'une activité : /fý ná/ « rapiécer », /fỵ fỵ ná nā/ «s'escrimer à rapiécer [un vêtement très usé] ». Elle peut également exprimer l'activité à laquelle réfère le verbe entendue dans un sens élargi, incluant tenants et aboutissants: /mé/ «enseigner», /mé mē/ « assurer la formation [de qqn]»; /ngw $\bar{\gamma} /$ «visiter, aller voir », $/ \mathrm{hj} \grave{\gamma} /$ 


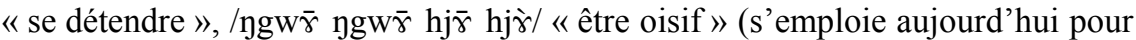
«faire du tourisme »). La forme rédupliquée est également attirée vers un sens figuré : / $\mathrm{p}^{\mathrm{h}}$ ว̀ « dénouer [un nœud] », $/ \mathrm{p}^{\mathrm{h}} \mathrm{\partial}^{\mathrm{h}} \mathrm{p}^{\mathrm{h}}$ ว̀/ « dénouer [une situation] » :
(1) $\operatorname{ts}^{\mathrm{h}} \overline{\mathrm{u}}$
Ş̄
$\mathrm{t}^{\mathrm{h}} \overline{\mathrm{u}}$
lē
$\mathrm{p}^{\mathrm{h}} \bar{\partial}^{\mathrm{r}} \mathrm{p}^{\mathrm{h}} \grave{\partial}^{\mathrm{c}}$
$\mathrm{m} \bar{\gamma} \mathrm{t}^{\mathrm{h}} \overline{\mathrm{v}}$.
DEICT. affaire
DeICT./THEM à nouveau
dénouer (DUPL.) NEG. parvenir

«Cette situation était inextricable.» (extrait d'un récit sur les suicides d'amoureux, autrefois très nombreux)

L'emploi d'une forme rédupliquée pour les sens figurés s'observe également pour les adjectifs : $/ \mathrm{t}^{\mathrm{h}} \mathrm{o} /$ « être adossé [à un arbre...] $) ; / \mathrm{t}^{\mathrm{h}} \overline{\mathrm{o}} \mathrm{t}^{\mathrm{h}} \mathrm{o} /$ « être frontaliers [se dit de deux pays, par exemple]». Lorsque leur sens s'y prête, les verbes rédupliqués prennent un sens réciproque: /lá/ «frapper» > lá lāa «se quereller », $/ \mathrm{k}^{\mathrm{h}} \overline{\mathrm{a}} /$ 《insulter » $>/ \mathrm{k}^{\mathrm{h}} \overline{\mathrm{a}} \mathrm{k}^{\mathrm{h}} \overline{\mathrm{a}} /$ « s'entr'insulter $», / \mathrm{su} \mathrm{u} /$ « connaître, savoir » > /sū sū̄/ « faire connaissance ; avoir une liaison ». Ce phénomène, présent dans d'autres langues, est rapporté par François 2004:184-185 à une valeur commune à toutes les réduplications de verbes, qui signaleraient un éclatement du procès. Cet éclatement peut être celui de deux ou plusieurs actants qui n'agissent pas à l'unisson, ou celui d'un procès qui ne s'oriente pas vers un accomplissement, se diluant en activité.

Deux exemples illustreront cette valeur de la réduplication. Dans un conte, une jeune fille qui n'arrive pas à descendre d'un arbre voit passer deux bergers, et tente d'attirer leur attention. Dans ce contexte, le verbe /ndə̀/ « appeler, crier » est rédupliqué en /ndə̄‘ ndə̀/; non pas action ponctuelle d'appeler, mais effort soutenu : « elle se mit à les appeler ». Le second exemple est tiré d'un récit sur les règles d'utilisation de l'eau des canaux de la ville de Lijiang avant l'arrivée de l'eau courante.

\begin{tabular}{|c|c|c|c|c|c|}
\hline (2) $t^{\mathrm{h}} \overline{\mathrm{u}}$ & ndzū & $\mathrm{gi}^{-}$ & $g \bar{v}$ & hó & nè \\
\hline repas du matin & manger & ACP./expérientiel & passer & après & INACC. \\
\hline sé, & hō phé & $\operatorname{ts}^{\mathrm{h}} \bar{\partial}^{\top} \mathrm{t} \mathrm{S}^{\mathrm{h}} \bar{\partial}^{\mathrm{c}}$ & $\mathrm{t}^{\mathrm{h}} \mathrm{a}$ & & sè. \\
\hline COND./THEM. & légumes & laver (DuPL.) & pouvoi & ê. autorisé & ACP. \\
\hline
\end{tabular}

«Quand on avait fini de manger le repas du matin, on pouvait laver ses légumes [dans le canal]»; en d'autres termes, l'heure était venue de laver les légumes. En employant la forme rédupliquée, l'énonciateur décrit l'association d'une activité à un certain moment de la journée.

Enfin, certaines formes sont lexicalisées (ce qui rejoint les observations de Hurch et Mattes, ce volume) : au verbe /má $\mathrm{m} \overline{\mathrm{a}} /$ 《construire, réparer» ne correspond pas de verbe monosyllabique /má/, même si l'on peut présumer que /má $\mathrm{mā} /$ provient de la réduplication d'un ancien /má/. De même, /tsē tsē/ «hacher de la viande» n'a plus de forme simplex; s'agissant d'un geste répétitif, on imagine sans peine le passage d'un monosyllabe souvent rédupliqué à un disyllabe. 
Dans le cas des adjectifs, les formes rédupliquées n'ont pas connu de spécialisation sémantique notable. Le sens le plus fréquent est celui de degré supérieur de : ainsi, $/ \mathrm{ts}^{\mathrm{h}} \mathrm{u} /$ 《 rapide » $>/ \mathrm{ts}^{\mathrm{h}} \overline{\mathrm{u}} \mathrm{t} \mathrm{s}^{\mathrm{h}} \mathrm{u} /$ « très rapide », valeur que l'on peut décrire en termes de recentrage sur la notion, d'identification au prototype de la qualité concernée. Le choix d'une forme rédupliquée, plutôt que d'un intensif du type " très, extrêmement », véhicule une nuance stylistique enjouée, affectueuse, le procédé ayant conservé quelque chose d'enfantin en dépit du corset phonologique étroit dans lequel il nous apparaît. (Au sujet de la nuance enfantine qui s'attache souvent à la réduplication, voir la présentation générale du présent volume, «Réduplication/redoublement : entre iconicité et système », qui souligne l'emploi fréquent de la réduplication dans le parler bébé.)

Signalons enfin que certains noms peuvent également être rédupliqués, avec le sens de « tous les... » : /dỳ/ « sol, terre », /dȳ dỳ/ « tous lieux; partout ». Les exemples ci-dessus appartiennent tous au dialecte de Lijiang, mais les phénomènes qu'ils illustrent sont présents dans tous les dialectes étudiés.

\subsection{Forme phonologique de la réduplication}

Le gabarit tonal du composé rédupliqué est entièrement prévisible d'après les tons de la forme simplex.

- A Lijiang, le comportement du ton dans la réduplication de monosyllabes $(\mathrm{A}>\mathrm{AA})$ est le suivant: le ton moyen se réduplique à l'identique (Moyen $>$ Moyen + Moyen), tandis que les tons Bas et Haut sont modifiés : Bas $>$ Moyen + Bas, Haut $>$ Haut + Moyen. Ce fait rapporté par plusieurs travaux antérieurs (He Jiren et Jiang Zhuyi 1985; He Zhiwu 1987:10-11; Guo Dalie, He Jiren et Yang Fuquan 1999:68-70) ressort clairement de la prononciation des informateurs, qui savent en outre confirmer l'identité des tons, par identification avec un mot de référence : ton de /lá/ « frapper », ton de $/ \overline{\mathbf{a}} /$ « tigre », ton de /là/ « main », ou ton de /là'/ « bougie ».

- A Fèng Kē, dans la réduplication de monosyllabes $(\mathrm{A}>\mathrm{AA})$, les tons Moyen et Haut se rédupliquent à l'identique (Moyen $>$ Moyen + Moyen, Haut $>$ Haut + Haut), tandis que les tons Bas et Bas-Moyen sont modifiés : le premier donne une séquence Moyen + Bas, et le second la séquence suivante : Moyen ( $\operatorname{sur}$ la $1^{\mathrm{e}}$ syllabe du composé rédupliqué) suivi de Bas-Moyen (sur la $2^{\mathrm{e}}$ syllabe).

Un grand nombre de disyllabes suivent le même schéma: dans ce cas (réduplication $\mathrm{AABB}$ ), les tons résultants sont les mêmes que si chacune des deux syllabes était rédupliquée séparément.

Dans un second ensemble, qui suit le schéma ABAB, un disyllabe est rédupliqué intégralement, tons compris. Ainsi, /ndá lā/ « de petite taille » donne /ndá lā ndá lā/ « de très petite taille », /lỵ fỵ́/ « tiède » donne /lỵ fỵ́ lỵ fỵ́/. Il peut s'agir du même simplex que dans le schéma AABB : ainsi, « très, très blanc »

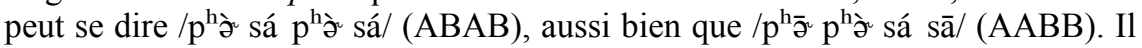
n'y a pas de différence sémantique qui ressorte entre l'un et l'autre schéma; le 
choix parait être une question d'habitudes personnelles. Les gabarits tonals sont identiques chez tous les informateurs interrogés, tandis que les phonèmes employés, et les nuances sémantiques, varient quelque peu. Ainsi, la formule désignant un vert intense, formée sur la base de /hə̀/, «vert-bleu», est prononcée, en fonction de l'informateur, /hà̀ zá hà zá/, /hò̀ zú hə̀̀ zú/, ou encore

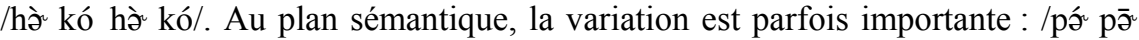
hwá hwā/ signifie dans le dialecte citadin «écrire et peindre », tandis que dans le village de /a s s̀̀/ / (网江金山乡文化行政村冷不罗自然村) l'expression a le sens « faire des graffitis (écrire sur les murs, etc.) ».

La différence phonologique entre les schémas $\mathrm{AABB}$ et $\mathrm{ABAB}$ est centrale dans le présent travail. Des faits phonologiques exposés ci-dessus, il ressort que si les syllabes rédupliquées ne sont pas en contact immédiat (schéma $A B A B$ ), aucune modification n'a lieu, tandis que si elles sont en contact immédiat (schémas $\mathrm{AA}$ et $\mathrm{AABB}$ ), certaines séquences de tons se trouvent modifiées.

L'orientation de la recherche présentée ici est suggérée par un constat phonétique : dans les disyllabes rédupliqués de forme $\mathrm{ABAB}$, où il n'y a pas de changement de catégorie tonale, la hauteur des tons des syllabes phonologiquement identiques est différente à l'oreille. (Dans ce qui suit, pour distinguer les réalisations phonétiques de ces quatre syllabes, elles seront désignées par A1 B1 A2 B2.) Lors de l'enquête, nos premières notations des tons résultant de la réduplication d'un disyllabe Bas + Haut oscillaient entre Bas + Haut + Bas + Moyen et Moyen + Haut + Bas + Moyen, avant que les informateurs ne nous reprennent, corrigeant en Bas + Haut + Bas + Haut.

\subsection{Hypothèse diachronique}

L'hypothèse à laquelle on est conduit est que tous les schémas observés en langue na pourraient provenir de réduplications à l'identique; les transformations ultérieures des séquences Haut + Haut en Haut + Moyen (qui n'a pas eu lieu en na de Fèng Kē, plus conservateur sur ce point), de Bas + Bas en Moyen + Bas, ainsi que de Bas-Moyen + Bas-Moyen en Moyen + BasMoyen à Fèng Kēe, ont en commun qu'elles tendent à établir un schéma mélodique descendant à l'échelle du composé rédupliqué, nivelant en tons Moyens les tons Bas en début de mot et les tons Hauts en fin de mot. Ces transformations constitueraient la phonologisation-la transformation en processus catégoriel - d'un phénomène phonétique attesté en synchronie: le marquage intonatif des frontières de mot. Le terme de «mot» est ici employé dans un sens extrêmement large. Le marquage des frontières se manifeste en réalité à l'échelle de divers groupements : mot lexical, syntagme, énoncé, et paragraphe oral. En naxi, le marquage de ces frontières se réalise par une descente progressive au cours du constituant concerné, et une descente plus forte tout à la fin du constituant (Michaud 2005:41-84). 
Cette hypothèse soulève la question des conditions historiques dans lesquelles la variation allophonique des tons aurait pu se trouver phonologisée : en effet, la variation est permanente dans les langues, et ne se fixe que dans des conditions particulières, déterminées par l'état du système linguistique (Martinet 2005 [1955]:3). La probabilité d'un changement dépend, plus que de la distance phonétique entre les allophones, de l'existence de facteurs qui, dans le système de la langue, déterminent le changement. L'hypothèse suggérée par Martine Mazaudon (communication personnelle) est que la langue na aurait connu, au cours de son histoire, une phase pendant laquelle elle aurait entamé une évolution vers un système prosodique à ton de mot; à ce stade, l'effet du marquage des frontières aurait joué à plein sur la réalisation phonétique du ton de mot, qui se serait étendu sur les deux syllabes de certains disyllabes. Pour prendre un exemple, la cible phonétique d'un ton bas n'aurait été atteinte que vers la fin de la seconde syllabe d'un disyllabe portant ce ton ${ }^{1}$. Par la suite, avant que l'évolution n'atteigne son terme (un système dans lequel un mot phonologique disyllabique ne peut porter qu'un seul ton, comme c'est le cas en tamang : voir Mazaudon 1973), elle se serait inversée, peut-être sous l'effet de langues influençantes. Lors du retour au système prosodique à un ton lexical par syllabe, chaque syllabe des disyllabes qui étaient passés au ton de mot se serait alors vu réassigner un ton propre; ce processus se serait fait par une réinterprétation dans le cadre proposé par le paradigme des trois principaux tons lexicaux, H, M et L. C'est alors que la différence phonétique de hauteur entre les deux syllabes des mots phonologiques en question aurait donné lieu à une réinterprétation. Tel serait le cas des schémas de réduplication de type AA et $\mathrm{AABB}$, mais aussi d'un certain nombre d'autres mots lexicaux : par exemple, « pois vert, petit pois », formé de /nỵ// « pois » et /hə̀/ " vert », serait un moment passé par un stade où le mot ne portait qu'un unique ton $\mathrm{L}$, dont la cible était atteinte tardivement; sa réalisation phonétique, proche de [nỵ hò], aurait ensuite été réinterprétée comme telle au plan phonologique (d'où la forme actuelle /nỵ hə̀/) lors du retour à un système « omnisyllabique » (selon la formule de James Matisoff, 1989), dans lequel chaque syllabe porte un ton lexical qui lui est propre.

Dans cette perspective, il devient compréhensible que l'effet du marquage des frontières de mots ait pu se trouver phonologisé. En effet, s'il a existé un

\footnotetext{
${ }^{1}$ Cette hypothèse, formulée en 2004, a récemment reçu un élément de confirmation : le système prosodique du dialecte na parlé sur le haut plateau de Yongning (丽江市宁蒗县 永宁乡), étudié sur place par le premier auteur d'octobre à décembre 2006, est par certains aspects proches d'un système de ton de mot. Un premier sondage, sur 500 mots, révèle qu'environ deux tiers des mots disyllabiques portent un schéma tonal $\mathrm{HH}, \mathrm{MM}$ ou LL, suggérant une modélisation en termes d'un unique ton, $\mathrm{H}, \mathrm{M}$ ou $\mathrm{L}$, se déployant sur le mot. Les autres combinaisons, HM, HL, MH, ML, LH, LM, sont attestées, mais ont une fréquence notablement plus basse. L'analyse de ces données est en cours, et se prolongera par l'extension de l'enquête à d'autres dialectes.
} 
stade de retour vers un système prosodique dans lequel chaque syllabe possède un ton propre, cela à partir d'un stade où certains mots ne possédaient plus qu'un ton pour le mot entier, le problème de la réinterprétation de la hauteur phonétique de chacune des syllabes d'un tel mot s'est posé de la façon suivante : les locuteurs ont effectué une réinterprétation catégorielle d'objets phonétiques au statut phonologique incertain. Telle est la situation que l'expérience mise en place ci-dessous vise à simuler, sans bien sûr prétendre reconstituer les conditions exactes de cette évolution.

\section{VOLET EXPERIMENTAL}

L'expérience menée vise à évaluer en synchronie la tension entre catégories phonologiques et variation allophonique des tons («variation allotonique »).

\subsection{Méthode : étude combinée de données de production et de perception}

La méthode exploratoire comporte deux volets complémentaires: une quantification, au plan de la production, des différences entre tons phonologiquement identiques dans le schéma $\mathrm{ABAB}$, et une étude de la perception de ces différences.

Huit locuteurs natifs du dialecte de Lijiang, âgés de 27 à 55 ans, ont enregistré les mêmes expressions rédupliquées.

Le premier paramètre étudié est la fréquence fondamentale, c'est-à-dire la fréquence de vibration des plis vocaux. Pour en obtenir une mesure très précise, et la compléter par une indication sur la qualité de voix, le quotient ouvert, l'enregistrement audio se double d'un enregistrement électroglottographique. L'électroglottographie est une technique non invasive de mesure de l'évolution de la surface d'accolement des plis vocaux à l'aide d'électrodes placées de part et d'autre du larynx (Fabre 1957). Un exposé détaillé sur cette technique, et sur la signification du quotient ouvert, est fourni par Henrich et al. 2004. En quelques mots : le quotient ouvert offre un indice du degré d'adduction des plis vocaux (Rothenberg et Mahshie 1988), la plage de variation allant d'environ $35 \%$ (voix très « pressée ») à 70\%, voix très « détendue » (Titze 1988:236).

Les autres mesures effectuées sont la durée et l'intensité acoustique.

Ce sont les données des deux premiers locuteurs qui ont été choisies pour le test de perception. Celui-ci a consisté à présenter les syllabes isolément (40 stimuli), puis par combinaisons de deux, explorant toute la combinatoire : $\mathrm{A} 1+\mathrm{B} 1, \mathrm{~A} 1+\mathrm{A} 2, \mathrm{~A} 1+\mathrm{B} 2, \mathrm{~B} 1+\mathrm{A} 1$, etc. (140 stimuli), et enfin par combinaisons de quatre (48 stimuli). Pour que l'attention des auditeurs se porte sur le ton, non sur les phonèmes ou les items lexicaux concernés, c'est le signal électroglottographique qui a été utilisé pour les tests, et non le signal audio : les auditeurs n'entendaient donc qu'une ligne mélodique portée par un bourdonnement. Les tests ont été soumis à 14 auditeurs, tous ayant le naxi de Lijiang pour langue maternelle. 


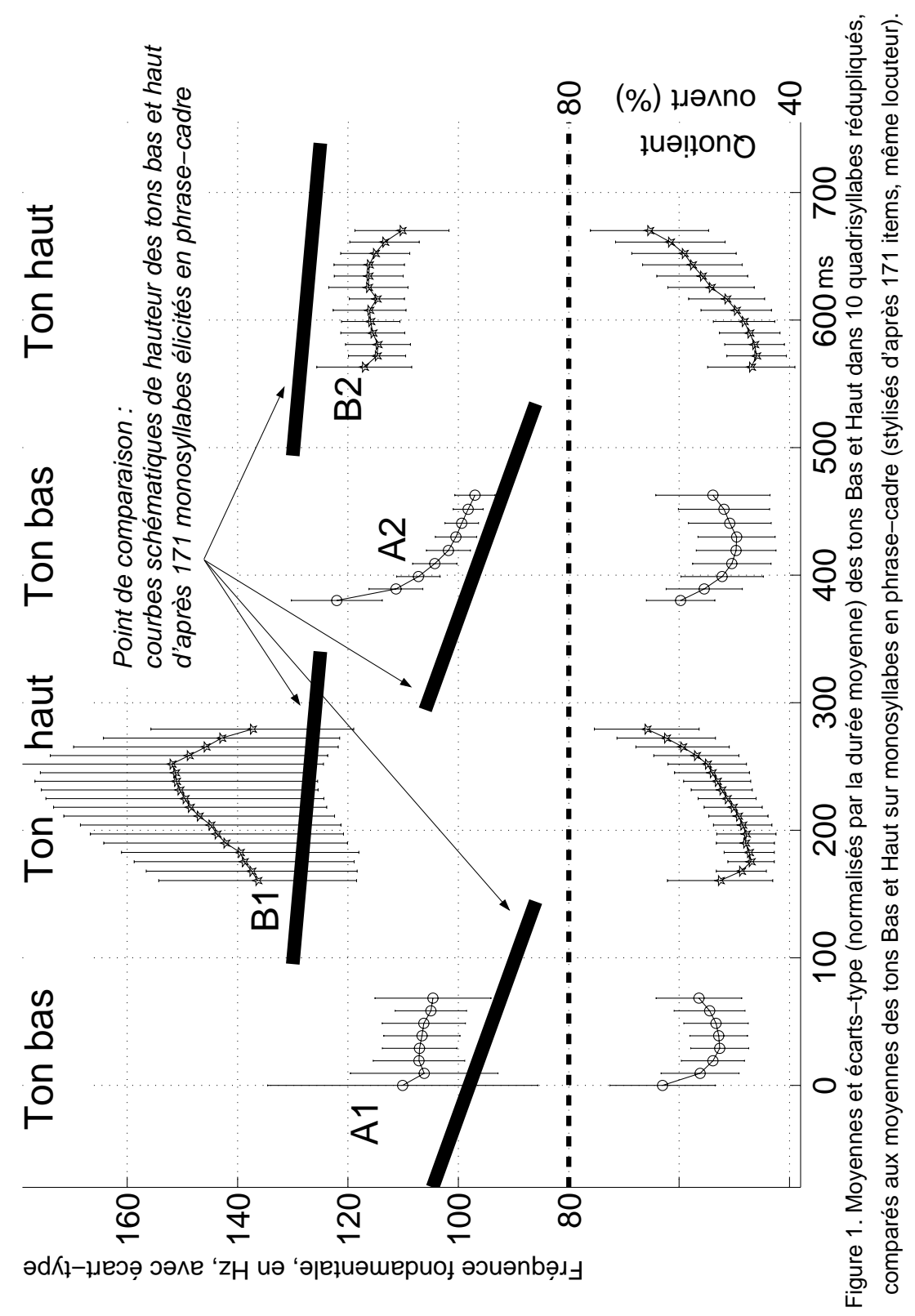




\subsection{Résultats des mesures sur les données de production}

La figure 1 présente, pour le premier des locuteurs, les moyennes des mesures sur 10 disyllabes rédupliqués de la forme $\mathrm{ABAB}$, ayant pour schéma tonal Bas + Haut + Bas + Haut : par exemple /hỳ zý hỳ zý/ « très, très rouge »;

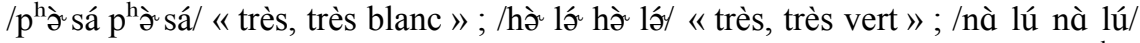
« très, très noir ». Ils sont formés d'un adjectif de couleur : /hỳ/ « rouge », / $\mathrm{p}^{\mathrm{h}}$ ว̀ « blanc ", /hə̀/ « vert / bleu ", /nà/ « noir », suivi d'un adjectif qui oriente vers une certaine nuance de couleur et de texture. La similarité de structure de ces items autorise à les moyenner. L'écart-type permet de visualiser la variation d'un item à l'autre ; les courbes schématiques des tons Bas et Haut tels qu'ils ont été réalisés à l'intérieur d'une phrase-cadre par le même informateur sont également reportées sur la figure (lignes épaisses), en vis-à-vis des syllabes concernées, offrant une valeur-repère ${ }^{2}$. La comparaison fait ressortir les faits suivants :

1) la fréquence fondamentale des syllabes $\mathrm{A} 1, \mathrm{~B} 1$ et $\mathrm{A} 2$ est significativement au-dessus des valeurs observées sur les monosyllabes en phrase-cadre, et B2 significativement au-dessous. (L'outil statistique employé est le test-t two-tail non apparié, appliqué sur les courbes rééchantillonnées. Les valeurs de p sont inférieures à 0.05 sur plus des $4 / 5^{\mathrm{e}}$ de la courbe dans tous les cas.)

2) A1 est plus élevé que A2, B1 plus élevé que B2.

En revanche, au plan de l'intensité acoustique globale, et de la qualité vocale (caractéristiques qualitatives de la phonation) telle qu'elle est reflétée par le quotient ouvert, aucune différence nette n'apparaît entre A1 et A2, B1 et B2 : la décroissance d'intensité de $\mathrm{A} 1$ à $\mathrm{A} 2$ et de $\mathrm{B} 1$ à $\mathrm{B} 2$ n'est que de l'ordre d'un décibel, et s'explique aisément par la différence de hauteur. Celle de quotient ouvert est également minime, et n'est pas significative (résultat du test- $t$ : $\mathrm{p}=0,40$ pour $\mathrm{A} 1 \sim \mathrm{A} 2, \mathrm{p}=0,66$ pour $\mathrm{B} 1 \sim \mathrm{B} 2$ ).

Ces faits se vérifient dans les données de production des sept autres locuteurs (présentées en intégralité dans Michaud 2005). Globalement, les réalisations des tons Bas et Hauts sont nettement différentes selon qu'ils se trouvent en première position $(\mathrm{A} 1, \mathrm{~B} 1)$ ou en deuxième position $(\mathrm{A} 2, \mathrm{~B} 2)$. Ces observations laissent à penser que les tons des paires de syllabes A1 A2 et B1 B2 pourraient, s'ils se trouvaient côte à côte, être perçus comme différents; dans le cadre des catégories tonales présentes en langue naxi, la différence phonétique entre ces paires de syllabes va dans le sens d'une évolution d'une séquence Haut + Haut

${ }^{2}$ Les courbes des monosyllabes en cadre sur lesquelles sont fondées les valeurs stylisées en lignes noires pleines sont publiées dans Michaud 2003. Les outils logiciels développés par A. Michaud (en collaboration) sont disponibles par l'intermédiaire de la page internet suivante : http://voiceresearch.free.fr/egg/ 
vers Haut + Moyen, d'une séquence Bas + Bas vers Moyen + Bas. Les tests de perception qui suivent visent à vérifier cette hypothèse.

\subsection{Résultat des tests de perception}

Les syllabes extraites des séquences A1 B1 A2 B2 ont d'abord été présentées isolément (rappel : dans tous les tests, les auditeurs n'entendaient pas le signal audio mais le signal électroglottographique, bourdonnement porteur de la ligne mélodique). Le tableau ci-dessous correspond aux données du premier locuteur, c'est-à-dire celles qui sont représentées sur la figure 1 .

Tableau 1. Résultat du test d'identification du ton des syllabes extraites des séquences rédupliquées A1 $\mathrm{B} 1$ A2 $\mathrm{B} 2.40$ stimuli (signal électroglottographique), 14 auditeurs.

\begin{tabular}{|l|l|l|l|l|l|l|}
\hline $\begin{array}{l}\text { Place dans } \\
\text { la forme } \\
\text { rédupli- } \\
\text { quée }\end{array}$ & $\begin{array}{l}\text { Ton } \\
\text { phono- } \\
\text { logique }\end{array}$ & $\begin{array}{l}\text { Ton perçu : \% de cas dans } \\
\text { lesquels le ton a été identifié } \\
\text { comme : } \\
\text { Haut }\end{array}$ & $\begin{array}{l}\text { Ton produit : } \mathrm{F}_{0} \text { moyen } \\
\text { (en Hz), et écart (en tons } \\
\text { musicaux) par rapport au } \\
\text { ton Moyen réalisé sur } \\
\text { monosyllabe isolé }\end{array}$ \\
\hline $1^{\mathrm{e}}(\mathrm{A} 1)$ & Bas & 14,5 & $\mathbf{6 6}$ & 19 & 0,5 & $106(+1 / 4$ de ton $)$ \\
\hline $2^{\mathrm{e}}(\mathrm{B} 1)$ & Haut & $\mathbf{8 2}$ & 10,5 & 0,5 & 7 & $145(+1$ ton $3 / 4)$ \\
\hline $3^{\mathrm{e}}(\mathrm{A} 2)$ & Bas & 3 & $\mathbf{6 5 , 5}$ & 31 & 0,5 & 103 (même hauteur) \\
\hline $4^{\mathrm{e}}(\mathrm{B} 2)$ & Haut & 45,5 & $\mathbf{4 7 , 5}$ & 4 & 3 & $115(+1$ ton) \\
\hline
\end{tabular}

Les deux tons Bas sont majoritairement perçus comme des tons Moyens $(66 \%)$; le premier ton Haut est identifié comme tel à $82 \%$, le second crée chez les auditeurs une hésitation entre Haut (45,5\%) et Moyen (47,5\%).

La deuxième partie du test, dans laquelle des disyllabes artificiellement reconstitués étaient soumis aux auditeurs, donne des résultats qui vont dans le même sens : la séquence A1 A2 est perçue comme portant deux tons Moyens (pourcentages : $51 \%$ de $\mathrm{M}$ pour $\mathrm{A} 1,62 \%$ de $\mathrm{M}$ pour $\mathrm{A} 2$ ), B1 B2 comme une séquence Haut + Moyen (pourcentages d'identification : $87 \%$ de Haut pour B1, $88 \%$ de Moyen pour B2). Enfin, dans la troisième partie du test, le quadrisyllabe d'origine, A1 B1 A2 B2, est majoritairement perçu comme une séquence Moyen + Haut + Bas + Moyen, c'est-à-dire que ses deux tons Bas sont perçus, le premier comme Moyen, le second comme Bas; et ses deux tons Hauts, le premier comme Haut, le second comme Moyen. Rappelons qu'à l'audition du signal audio, qui permet de reconnaître l'expression, d'accéder à son sens, les auditeurs attribuent unanimement à ces expressions une séquence tonale Bas + Haut + Bas + Haut. 


\subsection{Synthèse des résultats}

Conformément à l'hypothèse suggérée par l'examen des données de production (figure 1), la différence phonétique entre les deux tons Hauts (syllabes B1 et B2) est perçue par les auditeurs comme une différence catégorielle dans les conditions de l'expérience ; rappelons que dans les données de production, ces syllabes ne se trouvaient pas côte à côte. Cette différence catégorielle correspond précisément au schéma phonologique observé dans la réduplication AA (Haut donnant une séquence Haut + Moyen).

En revanche, les tons Bas (syllabes A1 A2) présentés côte à côte sont perçus comme une séquence Moyen + Moyen, et non Moyen + Bas. Notre interprétation est que les auditeurs retiennent le registre relativement élevé dans lequel ils sont tous deux réalisés (voir observation 1 ci-dessus), non la différence de hauteur entre les deux syllabes. Ceci amène à reconnaître que le protocole expérimental choisi ne fournit pas une simulation exacte des conditions d'évolution d'un composé rédupliqué Bas $>$ Bas + Bas : dans l'expérience, le premier ton Bas est en position initiale d'un quadrisyllabe, et le rehaussement qui en résulte est vraisemblablement supérieur à ce qui s'observerait en position initiale d'un disyllabe ; le second ton Bas n'est pas en position finale, et subit donc lui aussi un certain rehaussement. Le résultat confirme néanmoins l'intuition de départ selon laquelle la variation allophonique des tons peut, dans des conditions exceptionnelles, aller jusqu'à les faire percevoir comme des tons différents; selon l'hypothèse présentée ici, ce phénomène aurait eu lieu lorsque le système prosodique du naxi aurait connu un retour à un modèle tonal strictement syllabique (un ton lexical par syllabe) après une étape où il aurait entamé une transition vers un modèle à ton de mot.

\section{DISCUSSION ET CONCLUSION : TONS ET FRONTIERES INTONATIVES}

Une brève présentation des formes rédupliquées de langues typologiquement éloignées permettra de faire ressortir l'originalité des faits naxi.

La modification du ton d'une des syllabes dans les formes rédupliquées n'est pas particulière au naxi. Mais dans les langues africaines, plutôt que le schéma (3a), où la syllabe est rédupliquée avec son ton, c'est le schéma (3b) qui est couramment attesté : un même ton est associé aux deux syllabes identiques du composé rédupliqué. Nous notons, très classiquement, la syllabe par $\sigma$ et le ton lexical par $\mathrm{T}$.

(3a) $\sigma$

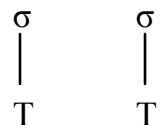

(3b)

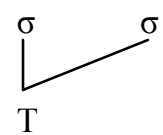


Ainsi, la forme expressive bambara [bòròbárá] (qui signifie : « de façon flasque ») est analysée par Dumestre 1987:95 comme porteuse d'un schéma Bas + Haut, chacun des tons se déployant sur une des moitiés de la forme rédupliquée :

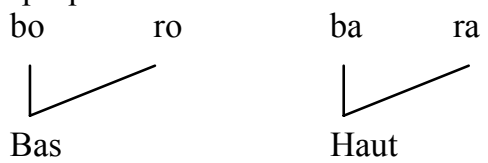

Phonétiquement, la cible, haute ou basse, est atteinte tardivement: sur la seconde syllabe, de sorte que le schéma Haut + Haut se réalise par une légère montée (notation informelle : HautHaut), le schéma Bas + Bas par une descente (notation informelle: BasBas). Au-delà des langues africaines, ce phénomène s'observe dans les séquences de syllabes basses du japonais (Rialland 2003).

En naxi, le schéma Bas + Bas se réalise également comme une descente, phonologisée en: Moyen + Bas; en revanche le schéma Haut + Haut, contrairement à ce que l'on attendrait sur le modèle « africain », ne se réalise pas comme montant mais comme descendant, phonologisé en : Haut + Moyen. La notation ( $3 b)$ ne paraît donc pas adéquate dans le cas du naxi. On est amené à préférer la notation (3a), qui conduit à l'hypothèse selon laquelle les schémas de réduplication par lesquels Haut donne une séquence Haut + Moyen et Bas une séquence Moyen + Bas ont leur origine, comme Moyen + Moyen, dans une réduplication à l'identique : Haut + Haut et Bas + Bas, qui aurait ensuite été érodée par des phénomènes intonatifs, à un stade où aurait existé une tendance à l'établissement d'un ton de mot; les contrastes tonals se seraient trouvés partiellement neutralisés sur certains mots, les mots très soudés que sont les composés rédupliqués étant parmi les premiers à connaître ce changement. Le ton Moyen s'en tire sans accrocs ; il paraît raisonnable d'attribuer cela au fait qu'il se trouve au centre de la plage de hauteur : la seule condition à sa bonne reconnaissance est qu'il n'atteigne pas les valeurs marquées qui caractérisent les tons Bas et Haut.

Ces observations autorisent une réflexion d'ordre général concernant la ligne de déclinaison. Le terme désigne la décroissance progressive de la fréquence fondamentale au cours de l'énoncé, notamment observée par Maeda (1976) en anglais. Elle peut être rapportée à une origine physiologique ; mais la diminution de la hauteur mélodique à mesure de l'énoncé n'est pas une décroissance mécanique : elle est intimement liée à la structuration énonciative (marquage des frontières, et hiérarchie de l'information, se traduisant par divers degrés de mise en valeur des mots par l'énonciateur). Selon les langues, la ligne de déclinaison joue un rôle plus ou moins important (Vaissière 1983, Michaud 2006a) : les langues telles que le naxi, le chinois, les langues romanes et germaniques en jouent abondamment, amplifiant ou annulant la déclinaison pour signaler le découpage en constituants (marquage des frontières, aussi appelé « intonation syntaxique », même si sa relation avec la syntaxe n'est pas bijective) et l'attitude 
de l'énonciateur, notamment le degré de prise en charge énonciative (voir Vaissière 2004). Ces observations rejoignent (nous semble-t-il) les travaux d'Abramson (1978, 1979), Gsell (1979), Luksaneeyanawin (1983) sur le thai, et de Chao Yuen-ren (1933), Hoa (1980), Shih Chilin (2000) sur le mandarin, qui conduisent ces chercheurs à distinguer clairement entre les tons lexicaux et les phénomènes intonatifs (supralexicaux). Les schémas de réduplication du naxi illustrent de façon exemplaire la superposition de divers ordres de phénomènes qui caractérise le domaine prosodique.

\section{CONCLUSION}

Les données phonétiques amènent à conclure que les deux ensembles observés en naxi, avec et sans changement de ton, ont une origine commune, dans un phénomène de réduplication totale. L'évolution qu'a connu le schéma $\mathrm{AA}$ (et $\mathrm{AABB}$ ) suggère que, dans des conditions phonologiques propices, des facteurs intonatifs peuvent modifier la réalisation de certains tons au point qu'ils soient réinterprétés comme une autre catégorie tonale.

L'analyse des faits naxi a une portée générale au plan de la modélisation de la prosodie, faisant ressortir la superposition de plusieurs ordres de phénomènes dans la courbe intonative.

Ces perspectives entraînent bien loin des questions de réduplication proprement dites, ce qui souligne précisément combien la réduplication peut être un angle d'approche utile pour comprendre les structures linguistiques.

\section{REMERCIEMENTS}

Vifs remerciements à Larry Hyman, Martine Mazaudon et Annie Rialland pour des discussions très éclairantes; à Vu-Ngoc Tuân pour son aide en programmation ; au Comité de rédaction, et en particulier à Catherine Chauvin ; aux partenaires du projet à Pékin (Huang Xing et Kong Jiangping) et sur le terrain: Guo Dalie, He Jiezhen, He Jiren, He Xueguang, Latami Dashi; au Centre de recherches sur la culture dongba et au Musée de la culture dongba de Lijiang ; et aux consultants et amis naxi, en particulier He Xixian et Mu Ruhua, et leur famille. Il va de soi que les auteurs sont seuls responsables d'éventuelles erreurs de fait et d'interprétation. 


\section{Bibliographie générale}

[La bibliographie est commune à l'ensemble des contributions au volume.

Version preprint.]

Abbi A., 1980, Semantic Grammar of Hindi, A Study in Reduplication, Delhi, Bahri Publications.

Abbi A., 1992, Reduplication in South Asian Languages. An Areal, Typological and Historical Study, New-Delhi, Allied Publishers.

Abbi A., 1997, Le redoublement dans les langues d'Asie du sud, Faits de langues, 10 , p. 31-36.

Abíódun M. A., 1997, More on reduplication in Yorùbá, Journal of Nigerian Languages and Literatures, 5, p. 93-98.

Abramson A. S., 1978, Static and dynamic acoustic cues in distinctive tone, Language and Speech, 21, p. 319-325.

Abramson A. S., 1979, The noncategorical perception of tone categories in Thai, in B. Lindblom \& S. Öhman (éds.), Frontiers of Speech Communication Research, Londres, Academic Press, p. 127-134.

Adelaar K. A., 2000, Siraya reduplication, Oceanic Linguistics, 39, 1, p. 33-52.

Adewole S. M., 1997, Reduplication in Yoruba, Linguistique Africaine, 18, p. $105-129$.

Ahenakew F. \& Wolfart H. C., 1983, Productive reduplication in Plains Cree, in W. Cowan (éd.), Actes du quatorzième congrès des Algonquinistes, Ottawa, Carleton University, p.369-377.

Allen J. P., 1984, The Inflection of the Verb in the Pyramid Texts, Malibu, Undena Publications, coll. Bibliotheca Aegyptia 2.

Allott A. \& Okell J., 2001, Burmese / Myanmar : A Dictionary of Grammatical Forms, Richmond (Surrey), Curzon Press.

Bach E., 1986, The algebra of events, Linguistics and Philosophy, 9, p. 5-16.

Bader F., 1968, Vocalisme et redoublement au parfait radical en latin, $B S L P, 63$, p. $160-196$.

Baker P., 2003, Reduplication in Mauritian Creole with notes on reduplication in Reunion Creole, in S. Kouwenberg (2003), p. 211-18.

Balusu R., 2005, Distributive reduplication in Telugu, Paper presented at NELS 36, U. Mass, Amherst. (http://semanticsarchive.net/Archive/mE0YTZkZ/balusuNELS.pdf)

Beedham C., 1982, The Passive Aspect in English, German and Russian, Tübingen, Günther Narr.

Bendahman J., 1993, Der reduplizierte Aorist in den indogermanischen Sprachen, Egelsbach / Cologne / New York, Hänsel-Hohenhausen [microfiches].

Bendjaballah S. \& Haiden M., 2003, Templatic Architecture, Recherches Linguistiques de Vincennes, 32, p. 157-168.

Bendjaballah S. \& Haiden M., à paraître, A typology of emptiness in templates, in J. Hartmann, V. Hegedus \& H. van Riemsdijk (éds.), Sounds of Silence, Amsterdam, Elsevier North-Holland.

Bernot D., 1980, Le Prédicat en birman parlé, Paris, Société d'études linguistiques et anthropologiques de France. 
Bernot D., 1983, Y a-t-il des catégories adjectivales et adverbiales en birman ?, Cahiers de l'Asie du Sud-Est, p. 67-78.

Bernot D., Cardinaud M.-H. \& Yin Yin Myint M., 2001, Grammaire birmane. Manuel du birman, vol. 2, Paris, L'Asiathèque - Langues et Mondes.

Björverud S., 1998, A Grammar of Lalo, Lund (Suède), Department of East Asian Languages, Lund University.

Blanchet A.-L.-P., 1850, Petit dictionnaire usuel de mimique et de dactylologie à l'usage des médecins et des gens du monde. Tome I, 2ème partie de La Surdi-Mutité, Paris, Chez Labé, Libraire-Editeur, réédition établie et présentée par Françoise Bonnal-Vergès, Limoges, Editions Lambert-Lucas, 2007.

Blust R., 1998, Ca-reduplication and Proto-Austronesian grammar, Oceanic Linguistics 37, 2, p. 29-64.

Blust R., 2001, Squib: Thao triplication, Oceanic Linguistics, 40, 2, p. 324-335.

Blust R., 2003, Thao Dictionary. Language and Linguistics Monograph Series, No. A5, Taipei, Institute of Linguistics (Preparatory Office), Academia Sinica.

Bolinger D. L. M., 1978, Intonation across languages, in Universals of Human Language, Stanford, Standford University Press, p. 471-524.

Bollée A., 2003, Reduplication in Seychelles Creole, in S. Kouwenberg (2003), p. 219-25

Bonnal F., 2004, Les Signes, à la lorgnette des Dictionnaires des XVIIIe et XIXe siècles, Surdités, 5/6, p. 16-59.

Bonnal-Vergès F., 2006a, Langue des signes française (LSF) : des lexiques des XVIII et XIXe siècles à la dictionnairique du XXIe siècle, in R. Sabria (éd.), Les langues des signes (LS) : recherches sociolinguistiques et linguistiques, Glottopol, 7, DYALANG, p. 160-190 (http://www.univ-rouen.fr/dyalang/glottopol/numero_7.html)

Bonnal-Vergès F., 2006b, Etymologie et langue des signes française (LSF), Etymologie, Bulag, 31, Université de Franche-Comté, p. 149-164.

Bonnal-Vergès F., à paraître en 2008, Sémiogenèse de la langue des signes française (LSF) : étude critique des signes attestés sur support papier depuis le XVIII siècle et nouvelles perspectives de dictionnaires (thèse de Doctorat en Sciences du Langage, Université Toulouse II, soutenue en décembre 2005), Limoges, éditions Lambert-Lucas.

Borghouts J. F., 1988, Aspectual Values of the Second Tenses in Middle Egyptian, in H. Altenmüller (éd.), Akten des Vierten Internationalen Ágyptologenkongresses in München 1983 [Studien zur Altägyptischen Kultur, supplément 3], vol. I, Hambourg, Helmut Buske, p. 29-42.

Brøndal V., 1948, Les parties du discours. Partes orationis. Etude sur les catégories linguistiques, Copenhague, Munksgaard.

Bybee J. L., Perkins R. D. \& Pagliuca W., 1994, The Evolution of Grammar. Tense, Aspect and Modality in the Languages of the World, Chicago/Londres, University of Chicago Press.

Chang, Laura M., 1998, Thao reduplication, Oceanic Linguistics, 37, 1, p. 277 297.

Chao Yuen-ren, 1933, Tone and intonation in Chinese, Bulletin of the Institute of History and Philology, 4, 3, p. 121-134.

Chao Yuen-ren, 1968, A Grammar of Spoken Chinese, Berkeley, University of California Press. 
Chaudenson R., 1974, Le Lexique du parler créole de la Réunion, Paris, Champion.

Choe J.-W., 1987, Anti-Quantifiers and a Theory of Distributivity, Ph.D. dissertation, GLSA. Amherst, University of Massachusets.

Chung S. \& Timberlake A., 1985, Tense, aspect, and mood, in T. Shopen (éd), Language Typology and Syntactic Description, Vol. III, Cambridge, Cambridge University Press, p. 202-258.

Comrie B., 1976, Aspect - An Introduction to the Study of Verbal Aspect and Related Problems, Cambridge, Cambridge University Press.

Conathan L., 2005, Arapaho verbal reduplication: form and meaning, in H. C. Wolfart (éd.), Papers of the Thirty-Sixth Algonquian Conference, Winnipeg, University of Manitoba, p. 19-34.

Cooper C. (éd.), 1979, $\Gamma \Delta \triangle \Gamma^{\circ} \cdot \nabla^{\natural} b \iota_{x}$ chî âimin weskach. Hard Times, Cree Publications, Mistissini, Canada.

Corbett G., 2000, Number, Cambridge, Cambridge University Press.

Culioli A., 1999, "Non mais, des fois!", in Pour une linguistique de l'énonciation, t. 3, Paris, Ophrys, p. 135-143.

Cusic D., 1981, Verbal Plurality and Aspect, PhD Dissertation, Stanford University.

Cuxac C., 2000a, Compositionnalité sublexicale morphémique-iconique en langue des signes française, Recherches linguistiques de Vincennes, "Langage et Surdité", 29, p. 55-72.

Cuxac C., 2000b, La Langue des Signes française, Faits de langues, 15/16, Paris/Gap, Ophrys.

Cuxac C., 2004, "Phonétique" de la LSF: une formalisation problématique, in Actes des Journées d'études internationales "La linguistique de la Langue des Signes Française: recherches actuelles" (23 et 24 septembre 2003), Cahiers du SILEX, Université Lille 3, p. 93-113.

Dahlstrom A., 1997, Fox (Mesquakie) reduplication, International Journal of American Linguistics, 63, 2, p. 205-226.

Damourette J. \& Pichon E., 1911-1940, Des mots à la pensée. Essai de grammaire de la langue française, Tome Sixième, Paris, Editions D'Artrey.

Danon-Boileau L. 1993, De quelques préjugés relatifs à l'usage des notions de motivation et d'iconicité, Faits de langues, 1, p. 79-87.

Davis B. L. \& MacNeilage P. F., 1990, Acquisition of correct vowel production: A quantitative case study, Journal of Speech and Hearing Research, 33, 1, p. 16-27.

DeLancey S., 1997, Grammaticalization and the gradience of categories. Relator nouns and postpositions in Tibetan and Burmese, in J. Bybee, J. Haiman \& S. Thompson (éds.), Essays on Language Function and Language Type: Dedicated to T. Givón, Amsterdam, John Benjamins, p. 51-69.

Denny J. P., 1989, The nature of polysynthesis in Algonquian and Eskimo, in D. B. Gerdts \& K. Michelson (éds.), Theoretical Perspectives on Native American Languages, Albany, NY, State University of New York Press, p. 230-258

Dik S., 1997, The Theory of Functional Grammar. Part 1: The Structure of the Clause [1ère éd. 1989], Berlin, Mouton de Gruyter.

Dillman A., 1907, Ethiopic Grammar, trad. par J. A. Crichton, Londres, Williams \& Norgate. [Orig. Grammatik der äthiopischen Sprache. 2d éd., rév. par C. Bezold, Leipzig, Tauchitz, 1899]. 
Dressler W. U., Dziubalska-Kolaczyk K., Gagarina N. \& Kilani-Schoch M., 2005, Reduplication in child language, in B. Hurch (2005), p. 455-474.

Dubois J., 1973, Dictionnaire de linguistique et des sciences du langage, Paris, Larousse.

Dubuisson C. (éd.), 1996, Grammaire descriptive de la LSQ, Le lexique (tome 2), Montréal, Université du Québec.

Dumestre G., 1987, Le bambara du Mali: essais de description linguistique, thèse de doctorat, Paris, Université de la Sorbonne Nouvelle/ Institut National des Langues et Civilisations Orientales.

Edel E., 1955-1964, Altägyptische Grammatik, Rome, Pontificium Institutum Biblicum

Edel E., 1959, Beiträge zur ägyptischen Grammatik. 1. Zum angeblich geminierenden Pseudopartizip der Verben III. infirmae, Zeitschrift für Agyptische Sprache und Altertumskunde, 84, p. 105-108.

Emeneau M. B., 1969, Onomatopoeics in the Indian Linguistic Area, Language, 45, 2, p. 294-99.

Fabre P., 1957, Un procédé électrique percutané d'inscription de l'accolement glottique au cours de la phonation: glottographie de haute fréquence, Bulletin de l'Académie Nationale de Médecine, 141, p. 66-69.

Fabricus A. H., 1998, A Comparative Survey of Reduplication in Australian Langages, Lincom Studies in Australian languages 03, Munich, Lincom Europa.

Fassi Fehri A. \& Vinet M.-T., 2004, Distribution of number and classifier in Arabic and Chinese and parametrization, Linguistic research, 9, 1, IERA Publications, Rabat, p. 6-51.

Ferguson C. A., 1983, Reduplication in child phonology, Journal of Child Language, 10 , p. $239-243$.

Ferrand J., ca 1780, Dictionnaire des sourds-muets, d'après l'édition du Dr J.A.A. Rattel, 1897, Paris, Baillière \& fils, réédition établie et présentée par Francoise Bonnal-Vergès, Limoges, Editions Lambert-Lucas, 2007.

Fischer S., 1973, Two processes of reduplication in American Sign Language, Foundations of Language, 9 , p. 469-480.

Fónagy I., 1983, La vive voix: essais de psycho-phonétique, "Langages et Sociétés", Paris, Payot.

François A., 2004, La réduplication en mwotlap: les paradoxes du fractionnement, Faits de langues, 23-24, Les langues austronesiennes, Paris, Ophrys, p. 177-194.

Frishberg N., 1975, Arbitrariness and Iconicity: Historical Change in American Sign Language, Language, 51, 3, p. 696-718.

García-Medall J., 2000, Sobre reduplicación morfológica en lenguas amerindias, in E. Ridruejo \& M. Fuertes (éds.), I Simposio Antonio Tovar sobre Lenguas Amerindias, Valladolid, Tordesillas.

Giambullari, 1551, De la lingua che si parla \& scrive in Firenze. Et uno dialogo di Giovan Batista Gelli sopra la difficultà dello ordinare detta lingua, Florence, Lorenzo Torrentino.

Gil D., 1988, Georgian reduplication and the domain of distributivity, Linguistics, 26, p. 1039-1065.

Gil D., 1993, Nominal and verbal quantification, Sprachtypologie und Universalien Forschung, 46, p. 275-317. 
Gil D., 1995, Universal Quantifiers and Distributivity, in E. Bach, E. Jelinek, A. Kratzer \& B. H. Partee (éds.), Quantification in Natural Languages, Kluwer Academic Publishers, Dordrecht, p. 321-362.

Gil D., 2005a, Distributive numerals, in M. Haspelmath, M. S. Dryer, D. Gil \& B. Comrie (éd.), The World Atlas of Language Structures, Oxford / New York, Oxford University Press, p. 222-225.

Gil D., 2005b, From Repetition to Reduplication in Riau Indonesian, in B. Hurch (2005), p. 31-64.

Girod M. \& al., 1990 et 1997, La Langue des Signes, tomes 2 et 3. Dictionnaire bilingue LSF / Français, Vincennes, Editions IVT.

Goldsmith J. A., 1979, Autosegmental Phonology, MIT, PhD.

Goldsmith J. A., 1990, Autosegmental \& Metrical Phonology, Oxford, Blackwell.

Gouffé C., 1975, Redoublement et réduplication en haoussa : formes et fonctions, BSLP, 70, p. 291-319.

Gsell R., 1979, La prosodie du thai standard: tons et accent, ms., Institut de Phonétique, Paris, Université Paris 3.

Guerssel M. \& Lowenstamm J., 1990, The Derivational Morphology of the Classical Arabic Verbal System, UQAM et Université Paris 7, ms.

Guo Dalie 郭大烈, He Jiren 和郎仁 \& Yang Fuquan 杨福泉, 1999, Naxi Wenhua Daguan (Présentation de la culture naxi) 纳西文化大观, Kunming 昆明, Yunnan Minzu Chubanshe 云南民族出版社.

Haag M., 1997, Continuous and discrete adjectival scales, Lingua, 103, p. 113126.

Hagège C. \& Haudricourt A.-G., 1978, La Phonologie panchronique, Paris, Presses Universitaires de France.

Haji-Abdolhosseini M., Massam D. \& Oda K., 2002, Number and events : Verbal reduplication in Niuean, Oceanic Linguistics, 41, 2, p. 475-492.

Hale K., 1983, Warlpiri and the grammar of non-configurational languages, Natural Language and Linguistic Theory, 1, p. 5-49.

Hammer F., 1997, Iconicité et réduplication en français, Folia Linguistica, 31, 34 , p. $285-300$.

Hashimoto M., 1970, Notes on Mandarin phonology, Studies in General and Oriental Linguistics presented to S. Hattori, Tokyo, The TEC Corporation, p. $207-220$.

Haudricourt A.-G., 1968, La langue de gomen et la langue de touho en Nouvelle-Calédonie, BSLP, 63, p. 218-235.

He Jiren 和即仁 \& Jiang Zhuyi 姜竹仪, 1985, Naxiyu Jianzhi (Présentation de la langue naxi) 纳西语简志, Beijing 北京, Renmin Chubanshe 民族出版社.

He Zhiwu 和志武, 1987, Naxiyu Jichu Yufa (Grammaire élémentaire du naxi) 纳西语基础语法, Kunming 昆明, Yunnan Minzu Chubanshe 云南民族出版社.

Heine B. \& Kuteva T., 2002, Word Lexicon of Grammaticalization, Cambridge, Cambridge University Press.

Helffer M., 1977, Les Chants dans l'Epopée tibétaine de Ge-sar d'après le livre de la Course de Cheval. Version chantée de Blo-bzang bstan-'dzin, Genève, Paris, Librairie Droz, Hautes Etudes Orientales, II.

Henrich N., d'Alessandro C., Castellengo M. \& Doval B., 2004, On the use of the derivative of electroglottographic signals for characterization of nonpathological voice phonation, Journal of the Acoustical Society of America, 115,3, p. $1321-1332$. 
Hjelmslev L., 1933, Structure générale des corrélations linguistiques, in Nouveaux Essais, Paris, Presses Universitaires de France, p. 25-66.

Hoa M., 1980, L'Accentuation en pékinois, thèse de doctorat d'Etat, Paris, Ecole des hautes études en sciences sociales.

Huáng Bùfán黄布凡, 1991 , 道孚语 Dàofúyü [la langue de Daofu], in Dài Qingxià \& al. (éds.), 藏缅语十五种 Zàngmiănyü shíwǔ zhǒng [15 langues tibéto-birmanes], Pékin, Beijing Yanshan Chubanshe 北京燕山出版社, p. 145.

Huang C.-R., 1992, Adjectival reduplication in Southern Min, Zhongguo jing nei yuyan ji yuyanxue, 1, Taïpei, Academia Sinica, p. 407-422.

Hurch B. (éd.), 2005, Studies on Reduplication, Berlin, Mouton de Gruyter.

Hurch B., 2002, Die sogenannte expletive Reduplikation im Tarahumara. Rehabilitation eines verpönten Begriffs : Euphonie, in M. Bommes, C. Noack \& D. Tophinke (eds.), Sprache als Form. Festschrift für Utz Maas zum 60. Geburtstag, Wiesbaden, Westdeutscher Verlag, p. 51-62.

Hurch B. \& Mattes V., 2004, Über die Entstehung von partieller Reduplikation, in Gertraud Fenk-Oczlon \& C. Winkler (éd.), Sprache und Natürlichkeit. Gedenkband für Willi Mayerthaler, Tübingen, Narr, p. 137-156.

Jacques G., 2004, Phonologie et morphologie du japhug (rGyalrong), thèse de troisième cycle, université de Paris 7. (http:// xiang.free.fr/these-japhug.pdf)

Jacques G. \& Chen Zhen 向柏箖陈珍, 2004, 茶堡话的重叠形式 Chábăohuà de chóngdié xíngshì [Les formes de réduplication en japhug], 民族语文 Mínzú yüwwén, 4, p. 7-11.

Jelinek E., 1984, Empty categories, case, and configurationality, Natural Language and Linguistic Theory, 2, p. 39-76.

Jensen C., 1989, O desenvolvimento histórico da língua Wayampi, Campinas, Brasil, Editora da Unicamp.

Jensen C., 1990, Cross-referencing changes in some Tupí-Guaraní languages, in D. Payne (éd.), Amazonian Linguistics, Studies in Lowland South American Languages, Austin, University of Texas Press, p. 117-158.

Jensen C., 1998, Comparative Tupí-Guaraní morpho-syntax, in D. Derbyshire \& G. Pullum (éds.), Handbook of Amazonian languages, Berlin, Mouton de Gruyter, IV, p. 490-603.

Junker M.-O., 1995, Syntaxe et sémantique des quantifieurs flottants tous et chacun : Distributivité en sémantique conceptuelle, Genève, Droz.

Junker M.-O., 1996, Comment quantifier en cri de l'est, Recherches amérindiennes au Ouébec, 26, 3-4, p. 13-19.

Junker M.-O., 2000, Quantification in East Cree and linguistic relativity [The Belcourt Lecture, delivered before the University of Manitoba on February 26th, 1999], Winnipeg, Voices of Rupert's Land.

Junker M.-O., 2002, East Cree relational verbs, International Journal of American Linguistics, 16, 52, p. 307-29.

Junker M.-O., 2004, Focus, Obviation and Word Order in East Cree, Lingua, 114,3 , p. $345-365$.

Junker M.-O. (réd.), 2000-2007, La toile de la langue crie de l'Est: Www.eastcree.org

Junker M.-O. \& Blacksmith L., 1994, Reduplication in East Cree, in W. Cowan (éd.), Actes du vingt-cinquième Congrès des Algonquinistes, p. 265-273.

Junker M.-O., MacKenzie M. \& Blacksmith L., 2000-2007, The Interactive East Cree Reference Grammar (Southern dialect), www.eastcree.org. 
Junker M.-O., MacKenzie M., Salt L., Duff A., Moar D. \& Salt R. (éds.), à paraître/ 2007, Le Dictionnaire du cri de l'Est de la Baie James sur la toile : français-cri et cri-français (dialectes du Sud et du Nord). http://dict.eastcree.org/

Jurafsky D., 1996, Universal tendencies in the semantics of the diminutive, Language, 72, 3, p. 533-578.

Kabore R., 1998, La réduplication, in S. Platiel \& R. Kabore (éds.), Les langues d'Afrique subsaharienne. Faits de langues, 11-12, Paris, Ophrys, p. 359-376.

Keenan E. L., 1985, Passive in the World's Languages, in T. Shopen (éd.), Language Universals and Syntactic Description. vol. I: Clause Structure, Cambridge, Cambridge University Press, p. 243-281.

Kerbrat-Orecchioni C., 2001, Oui, non, si : un trio célèbre et méconnu, Marges linguistiques, 2, p. 95-119. (http:/www.marges-linguistiques.com)

Kiparsky P., 1986, The Phonology of Reduplication, Université de Stanford, ms. Kiyomi S., 1995, A new approach to reduplication: a semantic study of noun and verb reduplication in the Malayo-Polynesian languages, Linguistics, 33, p. $1145-1167$.

Kouwenberg S. (éd.), 2003, Twice as Meaningful: Reduplication in Pidgins, Creoles and other contact languages, Londres, Battlebridge.

Kouwenberg S. \& LaCharité D., 2005, Less is more : Evidence from diminutive reduplication in Caribbean Creole languages, in B. Hurch (2005), p. 533-545.

Kröll H., 1991, Beitrag zu den Reduplikationen im Portugiesischen (II), Lusorama, 15, p. 25-44.

Laca B., sous presse, Pluralidad y aspecto verbal en español, Revista Española de Lingüística.

Langacker R, 1991, Noms et verbes, Communications, 53, Paris, Seuil, p. 103153.

Larson M., 2004, Distributive Numerals and Multi-verb Constructions in Baule, Legon-Trondheim Linguistics Project Annual Colloquium 2004, University of Ghana, Legon. (http://greece.imk.fhg.de/publications/Larson Coll04.pdf)

Leben W., 1978, The Representation of Tone, in V. Fromkin (éd.), Tone: A Linguistic Survey, New York, Academic Press.

Lee A. P., 2006, A Typological Study of Reduplication in Formosan Languages, Ph.D. dissertation, Colchester, University of Essex.

Leroy M. \& Morgenstern A., 2005, Reduplication before age two, in B. Hurch (2005), p. 475-491.

Li C. \& Thompson S., 1981, Mandarin Chinese. A Functional Reference Grammar, Berkeley, University of California Press.

Li P. J.-K., 1977, The internal relationships of Rukai, Bulletin of the Institute of History and Philology, 48, 1, p. 1-92.

Li P. J.-K., 1996, The pronominal systems in Rukai, in B. Nothofer (éd.), Reconstruction, Classification, Description: Festschrift in Honor of Professor Isidore Dyen, Hambourg, Abera Verlag, p. 209-230.

Li P. J.-K. \& Tsuchida S., 2001, Pazih dictionary, Language and Linguistics Monograph Series, No. A2, Taipei, Institute of Linguistics (Preparatory Office), Academia Sinica.

Li P. J.-K. \& Tsuchida S., 2006, Kavalan Dictionary, Language and Linguistics Monograph Series, No. A19, Taipei, Institute of Linguistics, Academia Sinica. 
Lillo-Martin D. \& Müller de Quadros R., sous presse, Focus constructions in ASL and LSB, in J. Quer (éd.), Signs of Time, Selected Papers of Theoretical Issues of Sign Language Research 2004, Hambourg, Signum Verlag.

Lin W.-Y., 2004, Vowel epenthesis and reduplication in Squliq and C?uli? Atayal dialects. Hsinchu, Taïwan, National Tsing Hua University MA thesis.

Lin Xiangrong 林向荣, 1993, 嘉戎语研究 Jiārongyü yánjiū [Etude sur la langue rGyalrong], Chengdu, 四川民族出版社 Sichuan minzu chubanshe.

Lín Yòu-jīng \& Luóěrwŭ (Nor-bu) 林幼菁、罗尔武, 2003, 茶堡嘉戎语大藏话的趋向前缀及动词词干变化 Chábăo jiāróngyü Dàzànghuà qūxiàng qiánzhuì [Les alternances de thèmes verbaux et les préfixes directionnels en japhug de Da-tshang], 民族语文 Mínzú yǚwén, 4, p. 19-29.

Lin Yòu-jīng, 2003, Tense and aspect morphology in the Zhuokeji rGyalrong verb, Cahiers de linguistique - Asie orientale, 32, 3, p. 245-286.

Loprieno A., 1995, Ancient Egyptian - A Linguistic Introduction, Cambridge, Cambridge University Press.

Lowenstamm J., 2003, A propos des gabarits, Recherches Linguistiques de Vincennes, 32, p. 7-30.

Lu S.-C., 2003, An Optimality Theory Approach to Reduplication in Formosan Languages, M.A. Thesis, Taipei, Taïwan, National Cheng-chi University.

Luksaneeyanawin S., 1983, Intonation in Thai, thèse de doctorat, University of Edinburgh.

Lutz V., 1953, Observations sur les affirmations, les négations et les réponses évasives dans la conversation du 20 ème siècle. Thèse, Université de Zurich.

MacKenzie M., Junker M.-O., Salt L., Duff E., Moar D., Salt R., Neeposh E. \& Jancewicz B. (éds), 2004-2006, The Eastern James Bay Cree Dictionary on the Web : English-Cree and Cree-English (Northern and Southern dialects). (http://dict.eastcree.org/)

Maeda S., 1976, A Characterization of American English Intonation, thèse de doctorat, Cambridge, Massachusetts, M.I.T.

Malone J. L., 1997, On reduplication in Ojibwa, Anthropological Linguistics, 39,3, p. 437-458.

Marantz A., 1982, Re Reduplication, Linguistic Inquiry, 13, 3, p. 435-482.

Martinet A., 1980, Eléments de linguistique générale, Paris, Armand Colin.

Martinet A., 2005, Economie des changements phonétiques: traité de phonologie diachronique, Paris, Maisonneuve \& Larose. (1ère éd. : 1955.)

Mathieu B., 2004, La distinction entre Textes des Pyramides et Textes des Sarcophages est-elle légitime?, in S. Bickel \& B. Mathieu (éds.), D'un monde à l'autre: textes des Pyramides et textes des Sarcophages. Actes de la table ronde internationale "Textes des Pyramides versus Textes des Sarcophages". IFAO, 24-26 septembre 2001, Le Caire, Institut Français d'Archéologie Orientale, coll. Bibliothèque d'étude, p. 247-261.

Matisoff J. A., 1973, The Grammar of Lahu, Berkeley, University of California Press (Linguistics), vol. 75.

Matisoff J. A., 1989, Tone, intonation, and sound symbolism in Lahu : loading the syllable canon, Linguistics of the Tibeto-Burman Area, 12, 2, p. 147-163.

Matisoff J. A., 1990, On Megalocomparison, Language, 66, 1, p. 106-120.

Mattes V., 2007, Types of Reduplication. A Case Study of Bikol, PhD dissertation, Université de Graz.

Mawet F., 1993, Présents athématiques à redoublement : une catégorie héritée de l'indo-européen?, BSLP, 88, p. 85-101. 
Mayerthaler W., 1977, Studien zur theoretischen und französischen Morphologie." Reduplikation, Echowörter, morphologische Natürlichkeit, Haplologie, Produktivität, Regeltelescoping, paradigmatischer Ausgleich, Tübingen, Niemeyer.

Mazaudon M., 1973, Phonologie tamang: étude phonologique du dialecte tamang de Risiangku, langue tibéto-birmane du Népal, Paris, Société d'études linguistiques et anthropologiques de France.

McCarthy J., 1979, Formal Problems in Semitic Phonology and Morphology, New York, Garland Press.

McCarthy J., 1981, A Prosodic Theory of Nonconcatenative Morphology, Linguistic Inquiry, 12, p. 373-418.

Mel'cuk I., 2000, Cours de morphologie générale (théorique et descriptive), Presses de l'Université de Montréal, CNRS Editions.

Mgon po dbang rgyal, 2004, Zlos sgra'i tshig mdzod (Dictionnaire des formules rédupliquées), Beijing, Mi rigs dpe skrun khang.

Michailovsky B., 1988, La langue hayu, Paris, Editions du CNRS.

Michailovsky B. \& Michaud A., 2006, Syllabic inventory of a Western Naxi dialect, and correspondence with Joseph F. Rock's transcriptions, Cahiers de linguistique - Asie Orientale, 35, 1, p. 3-21.

Michaud A., 2003, Focus et tons lexicaux: de la variété des paramètres prosodiques, in A. Lacheret-Dujour \& J. François (éds.), Fonction et moyens d'expression de la focalisation à travers les langues, Mémoires de la Société de Linguistique de Paris, nouvelle série, tome XIII, Louvain, Peeters, p. 1347.

Michaud A., 2005, Prosodie de langues à tons (naxi et vietnamien), prosodie de l'anglais : éclairages croisés, thèse de doctorat, département de Sciences du langage, Paris, Université Paris 3 - Sorbonne Nouvelle. Disponible en ligne à l'adresse suivante : http://tel.archives-ouvertes.fr/tel-00130149

Michaud A., 2006a, Replicating in Naxi (Tibeto-Burman) an experiment designed for Yorùbá : An approach to 'prominence-sensitive prosody' vs. 'calculated prosody', Actes de la conférence internationale Speech Prosody 2006, Dresde, p. 819-822.

Michaud A., 2006b, Tonal reassociation and rising tonal contours in Naxi, Linguistics of the Tibeto-Burman Area, 29, 1, p. 61-94.

Migge B., 2003, The origin of predicate reduplication in Suriname Maroon Creole, in S. Kouwenberg (2003), p. 61-71.

Mignot L., 1980, Modes d'expression en vietnamien et en birman à travers la littérature contemporaine. Comparaison au niveau du fond et de la forme. Thèse de Doctorat de 3e cycle en Anthropologie Sociale et Culturelle, Paris, INALCO.

Miller C., 2000, Regards sur la phonologie des langues signées, Recherches linguistiques de Vincennes, "Langage et Surdité", 29, p. 101-120.

Montaut A., 2004, Hindi Grammar, Munich, Lincom-Europa.

Moravcsik E. A., 1978, Reduplicative Constructions, in J. H. Greenberg (éd.), Universals of Human Language, Vol. 3: Word Structure, Stanford, Stanford University Press, p. 297-334.

Moskowitz A. I., 1973, Acquisition of phonology and syntax: a preliminary study, in Approches to Natural Language, J. Hintikka, E. A. Moravesik \& P. Suppes, Dordrecht, Reidel, p. 48-84. 
Mourelatos A. P. D., 1981, Events, Processes, and States, in P. Tedechi \& A. Zaenen (éds.), Tense and Aspect, New York, Academic Press, coll. Syntax and Semantics, p. 191-212.

Naga Acharya Sangye T. \& Tsepak R., 1994, Bod dbyin shan sbyar gyi tshig tshogs dang gtam dpe. Tibetan Quadrisyllabics, Phrases and Idioms, Dharamsala, Library of Tibetan Works and Archives.

Nève F.-X., 1996, Essai de Grammaire de la Langue des Signes Française, Fascicule CCLXXI, Librairie Droz, Genève.

Newman P., 1980, The Classification of Chadic within Afroasiatic, Leiden, Universitaire Pers Leiden.

Newman P., 1990, Nominal and Verbal Plurality in Chadic, Dordrecht, Foris, coll. Publications in African Languages and Linguistics.

Niepokuj M., 1997, The Development of Verbal Reduplication in IndoEuropean, Journal of Indoeuropean Studies Monograph, 24, Washington, Institute for the Study of Man.

Oliveri F., 2000, La Gestualità dei Siciliani, Palerme, Krea.

Ozanne-Rivierre F., 1986, Redoublement expressif et dédoublement des séries consonantiques dans les langues des îles Loyauté (Nouvelle-Calédonie), in C. Corne \& A. Pawley (éds.), Le coq et le cagou : Essays on French \& Pacific Languages in Honour of Jim Hollyman [Te Reo], 29, p. 25-53.

Paris M.-C., 1981, Problèmes de syntaxe et de sémantique en linguistique chinoise, Paris, Collège de France.

Paris M.-C., 1995, Type de prédication et copie du verbe en chinois standard, réimpr. in M.-C. Paris, Linguistique chinoise et linguistique générale, 2003, Paris, L'Harmattan, p. 51-69.

Paul H., 1880, Prinzipien der Sprachgeschichte, Halle, Max Niemeyer.

Peust C., 1999, Egyptian Phonology, Göttingen, Peust \& Gutschmidt, coll. Monographien zur Ägyptischen Sprache.

Pfau R. \& Steinbach M., 2004, Backward and sideward reduplication in German Sign Language, in B. Hurch (2005), p. 569-594.

Pott A., 1862, Doppelung (Reduplikation, Gemination) als eines der wichtigsten Bildungsmittel der Sprache, beleuchtet aus Sprachen aller Welttheile, Lemgo, Detmold, Meyer. (http://www-gewi.uni-graz.at/ling/proj/redup/pott/)

Procházka S., 1995, Semantische Funktionen der reduplizierten Wurzeln im Arabischen, in Archiv Orientalni, 63, p. 39-70.

Proulx P., 2005, Reduplication in Proto-Algonquian and Proto-CentralAlgonquian, International Journal of American Linguistics, 71, 2, p. 193214.

Pulleyblank D., sous presse/ 2007, Patterns of reduplication in Yoruba, in K. Hanson \& S. Inkelas (éds.), The Nature of the Word: Essays in Honor of Paul Kiparsky, Cambridge, Mass., MIT Press.

Qú Aitáng 篗靄堂, 1983, 嘉线语动词的人称范畴 Jiāróngyü dòngcí de rénchēng fànchóu [La catégorie de la personne dans le système verbal de langue rGyalrong], 民族语文 Mínzú yǘwén, 4, p. 35-60.

Quack J. F., 2003, Zum Charakter der "zweiradikaligen" Verben des Ägyptischen, in M. L. Bender, D. Appelyard, M. Lamberti \& G. Takács (éds.), Afrasian : Selected Comparative-Historical Studies in Memory of Igor M. Diakonoff, Munich, Lincom, coll. Lincom Studies in Afro-Asiatic Linguistics, p. 167-174. 
Regier T., 1998, Reduplication and the Arbitrariness of the Sign, Proceedings of the Twentieth Annual Conference of the Cognitive Science Society, Lawrence Erlbaum Associates, Mahwah, New Jersey, p. 887-892.

Reintges C. H., 1994, Egyptian Root-and-Pattern Morphology, Lingua Aegyptia, 4, p. 213-244.

Reintges C. H., 1997, Passive Voice in Older Egyptian - A Morpho-Syntactic Study, La Haye, Holland Academic Graphics, coll. HIL Dissertation Series.

Reintges C. H., 2003, The Reduplicative Passive in Older Egyptian : Description and Typology, in M. L. Bender, D. Appelyard, M. Lamberti \& G. Takács (éds.), Afrasian: Selected Comparative-Historical Studies in Memory of Igor M. Diakonoff, Munich, Lincom, coll. Lincom Studies in Afro-Asiatic Linguistics, p. 175-186.

Reintges C. H., 2004a, The Older Egyptian $s \underline{d} m(. w)=f$ Passive Revisited, Folia Orientalia, 40, p. 51-70.

Reintges C. H., 2004b, Coptic Egyptian (Sahidic dialect), A Learner's Grammar, Cologne, Rüdiger Köppe, coll. Afrikawissenschaftliche Lehrbücher 15.

Rialland A., 2003, Comments on Yasuko Nagano-Madsen : Phonetic realization of the HL and LH accents in Japanese, in S. Kaji (éd.), Cross-linguistic studies of tonal phenomena: historical developments, phonetics of tone, Tokyo, Research Institute for Languages and Cultures of Asia and Africa, Tokyo, University of Foreign Studies, p. 265-272.

Risler A \& Lejeune F, 2003, Les configurations manuelles pronominales en LSF, essai de classification, in Actes des Journées "Recherches sur les langues signées" (23 et 24 novembre 2001), Toulouse, Presses Universitaires Toulouse-Le Mirail, p. 103-121.

Risler A., 2000, La Langue des Signes Française, langue iconique. Thèse de doctorat, Université Toulouse le Mirail.

Risler A., 2003, Point de vue cognitiviste sur les espaces créés en langue des signes : espace lexical, espace syntaxique, Lidil, 26 "Gestualité et syntaxe", Université Stendhal, Grenoble, p. 45-61.

Risler A., 2006, La simultanéité dans les signes processifs, in R. Sabria (éd.), Les langues des signes (LS): recherches sociolinguistiques et linguistiques, Glottopol, 7, DYALANG, p. 53-71 (http://www.univ-rouen.fr/dyalang/glottopol/numero 7.html)

Rivierre J.-C. \& Ozanne-Rivierre F., 2004, Evolution des formes canoniques dans les langues de Nouvelle-Calédonie, Faits de langues, 23-24, p. 141-153.

Rodrigues A., 1953, Morfologia do verbo Tupi, Letras (Separata n ${ }^{\circ}$ ), p. 121152.

Rose F., 2003, Morphosyntaxe de l'émérillon. Une langue tupi-guarani de Guyane française, Thèse de Doctorat en Sciences du Langage, Université Lumière Lyon II.

Rose F., 2005, Reduplication in Tupi-Guarani languages : going into opposite directions, in B. Hurch (2005), p. 351-368.

Rothenberg M. \& Mahshie J. J., 1988, Monitoring vocal fold abduction through vocal fold contact area, Journal of Speech and Hearing Research, 31, p. 33851.

Rousseau A., 2002, La pluralisation nominale et verbale : étude sémantique et syntaxique dans une perspective typologique, in Mémoires de la Société de Linguistique de Paris, Vol. XII: La pluralite, p. 23-43. 
Rubino C., 2005, Reduplication: form, function and distribution, in B. Hurch (2005), p. 11-29.

Safir K. \& Stowell T., 1988, Binominal Each, in J. Blevins \& J. Carter (éds.), Proceedings of the Northeast Linguistic Society, volume 18, Amherst, GLSA University of Massachusetts, p. 426-450.

Sapir E., 1921, Language, New York, Harcourt, Brace \& Company.

Schembri A., 2001, Issues in the Analysis of Polycomponential Verbs in Australian Sign Language (Auslan), Thèse de Doctorat, University of Sydney.

Schuh R., 2002, The Locus of Pluractional Reduplication in West Chadic, UCLA, ms.

Schultze-Berndt E., 2000, Simple and Complex Verbs in Jaminjung : a Study of Event Categorisation in an Australian Language, Nimègue/Wageningen, MPI Series in Psycholinguistics 14.

Seki L., 2000, Gramática do Kamaiurá, Campinas, Editora da Unicamp.

Shih Chilin, 2000, A Declination Model of Mandarin Chinese, in A. Botinis (éd.), Intonation: Analysis, Modelling and Technology, Dordrecht, Kluwer Academic Publishers, p. 243-268.

Shukla S., 2001, Hindi Morphology, LINCOM Studies in Indo-European Linguistics 15, Munich, Lincom-Europa.

Sicard R.-A.-C., 1808, Théorie des Signes ou Introduction à l'étude des langues où le sens des mots au lieu d'être défini est mis en action, Tomes 1 et 2, Paris, Dentu \& Delalain.

Siewierska A., 1984, The Passive. A Comparative Linguistic Analysis, Londres, Croom Helm.

Singh R., 2005, Reduplication in Modern Hindi and the Theory of Reduplication, in B. Hurch (2005), p. 263-81.

Skoda F., 1982, Le Redoublement expressif, un universel linguistique : analyse du procédé en grec ancien et en d'autres langues, Paris, Société d'études linguistiques et anthropologiques de France.

Smith C. S., 1991, The Parameter of Aspect, Dordrecht, Kluwer, coll. Studies in Linguistics and Philosophy.

Srichampa S., 2002, Vietnamese Verbal Reduplication, in R. S. Bauer (éd.), Collected Papers on Southeast Asian and Pacific Languages, Canberra, Australian National University, Research School of Pacific and Asian Studies.

Stark R. E., 1986, Prespeech segmental feature development, in P. Fletcher \& M. Garman (éds.), Language Acquisition, New York, Cambridge University Press, p. 149-173.

Stokoe W., 1960, Sign Language structure: an outline of the visual communication systems of the American deaf, Studies in Linguistics, Occasional Papers, 8, University of Buffalo, Buffalo.

Strauss S., 2002, Distinctions in completives : The relevance of resistance in Korean $V$-a/e pelita and V-ko malta and Japanese V-te shimau, Journal of Pragmatics, 34, p. 143-166.

Sun T.-S. J. 孙天心 \& Shídānluó (bsTan 'dzin blo) 石丹罗，2004， 草登嘉线语的状貌词 Cǎodēng jiāróngyü de zhuàngmàocí [Les idéophones en rgyalrong de Tshobdun], 民族语文 Mínzú yüwén, 5, p. 1-11.

Sun T.-S. J., 1998, Nominal morphology in Caodeng rGyalrong, The Bulletin of the Institute of History and Philology, Vol. 69, 1, p. 103-149. 
Sun T.-S. J., 2003, Caodeng rGyalrong, in G. Thurgood \& R. J. LaPolla (éd.), Sino-Tibetan Languages, Londres, Routledge, p. 490-502.

Supalla T. \& Newport E., 1978, How many seats in a chair? The derivation of nouns and verbs in ASL, in P. Siple (éd.), Understanding Language through Sign Language Research, New York, Academic Press, p. 91-132.

Talmy L., 1985, Lexicalization patterns : semantic structure in lexical forms, in T. Shopen (éd.), Language Typology and Syntactic Description III, Cambridge, Cambridge University Press, p. 57-149.

Titze I. R., 1988, Regulation of vocal power and efficiency by subglottal pressure and glottal width, in O. Fujimura (éd.), Vocal Fold Physiology: Voice Production, Mechanisms and Functions, New York, Raven Press, p. 227-237.

Tournadre N. \& Dorje S., 2002, Manuel de tibétain standard. Langue et civilisation [1ère éd. 1998], Paris, L'Asiathèque - Langues et Mondes.

Tseng M., 2003, Reduplication as Affixation in Paiwan, M.A. Thesis, Chiayi, Taïwan, National Chung-cheng University.

Uray G., 1955, Duplication, Gemination and Triplication in Tibetan, Acta Orientalae Hungaricae (IV), fasc. 1-3, p. 177-244.

Vacek J., 1989, On Semantic Reduplication in Indian Languages : In the Margin of Language Universals, Archiv Orientalni, 57, p. 347-58.

Vaissière J., 1983, Language-independent prosodic features, in A. Cutler \& R. Ladd (éds.), Prosody: Models and Measurements, Berlin, Springer Verlag, p. 53-66.

Vaissière J., 2004, The Perception of Intonation, in D. B. Pisoni \& R. E. Remez (éds.), Handbook of Speech Perception, Oxford, G.B. I Cambridge, Massachusetts, Blackwell Publishers, p. 236-263.

Van Geenhoven V., 2004, For-adverbials, frequentative aspect and pluractionality, Natural Language Semantics, 12, p. 135-190.

Vendler Z., 1967, Linguistics and Philosophy, Ithaca, Cornell University Press.

Vernus P., 1996, La position linguistique des Textes des Sarcophages, in H. Willems (éd.), The World of the Coffin Texts. Proceedings of the Symposium held on the Occasion of the 100th Birthday of Adriaan de Buck, Leiden 17-19 December 1992, Louvain, Peeters, coll. Egyptologische uitgaven, p. 143-196.

Vittrant A., 2004, La modalité et ses corrélats en birman, dans une perspective comparative, Thèse de Doctorat en Sciences du Langage, Université Paris VIII (Vincennes / Saint-Denis).

Vittrant A., 2005, Classifier systems and noun categorization devices in Burmese, in Proceedings of the 28th Annual Meeting of the Berkeley Linguistics Society (BLS) 28, Berkeley, Berkeley Linguistics Society, p. 129148.

Wilbur R., 1973, The Phonology of Reduplication, Bloomington, Indiana University Linguistics Club.

Wilbur R., 2004, A reanalysis of reduplication in ASL, in B. Hurch (2005), p. $595-623$.

Wolfart H. C., 1973, Plains Cree: A Grammatical Study, American Philosophical Society Transactions, numéro spécial, 63, Philadelphie, American Philosophical Society.

Wood E, 2002, Plurality of events and event-phases, SALT 12 Conference, San Diego. http://ling.ucsd.edu/events/salt/salt12.wood.pdf 
Woodward J. C. Jr., 1976, Signs of Change : Historical Variation in ASL, Sign Language Studies, 10, p. 81-94.

Yeh Shih-Chi, 2003, Syllabification and Reduplication in Amis, MA thesis, Hsinchu, Taïwan, National Tsing Hua University.

Yip M., 1982, Reduplication and C-V skeleta in Chinese secret languages, Linguistic Inquiry, 13, 4, p. 637-661.

Zeitoun E., 1995, Problèmes de linguistique dans les langues aborigènes de Taiwan, Thèse de 3ème cycle, Paris, Université Denis Diderot Paris 7.

Zeitoun E., 2004. Typologie des langues austronésiennes de Taïwan, Faits de Langues : Les langues austronésiennes, 23/24, Paris/ Gap, Ophrys, p. 41-58.

Zeitoun E., à paraître, A Grammar of Mantauran Rukai, Language and Linguistics Monograph Series A4/2, Taipei, Institute of Linguistics, Academia Sinica.

Zeitoun E. \& Wu Chen-huei, 2005, Saisiyat reduplication revisited, Concentric, 31, 2, Taipei, Taïwan, National Taiwan Normal University, p. 31-56.

Zeitoun E. \& Wu Chen-huei, 2006, An overview of reduplication in Formosan languages, Festschrift in honor of Prof. Paul Jen-kuei Li on His Seventieth Birthday, Language and Linguistics Monograph Series, Taipei, Institute of Linguistics, Academia Sinica.

Zhang Liansheng, 1985, The Phonetic Structure of ABCB Type Words in Lhasa Tibetan, in B. N. Aziz \& M. Kapstein (éds.), Soundings in Tibetan Civilisation, New Delhi, Manohar, p. 20-34

Zhang Yisun \& al., 1996, Bod rgya tshig mdzod chen mo (Grand dictionnaire bilingue tibétain-chinois), Beijing, Mi rigs dpe skrun khang (Presses des Nationalités). 